\title{
Polynomial Psychophysics of Risk
}

\author{
Clyde H. Coombs and Lily C. Huang ${ }^{1}$
}

University of Michigan, Ann Arbor, Michigan 48104

\begin{abstract}
Three mathematical transformations on two-outcome games are defined. It is assumed that these transformations induce corresponding transformations on perceived risk. The rule governing the joint effect of these transformations is assumed to be the distributive model. An experiment is reported in which a class of simple polynomials are compared using the measurement-free methods of polynomial conjoint measurement. Substantial support for the distributive model is obtained.
\end{abstract}

\section{InTRODUCTION}

Portfolio theory (Coombs and Huang, 1968; Coombs and Meyer, 1968) is a theory of risk preference and is intrinsically independent of the definition of risk. Experimental study of portfolio theory, however, is dependent upon the manipulation of risk and hence discrepant experimental results do not clearly distinguish between the faults of portfolio theory and the incorrectness of assumptions about the nature of risk. In this paper we approach the problem of risk itself, independently of the problem of risk preference.

There are in the literature various attempts to define risk for purposes of descriptive theory and normative theory (e.g., Royden, Suppes, and Walsh, 1959; Coombs and Pruitt, 1960, 1961; Markowitz, 1959). Some experimental studies of the descriptive approaches have been made and the results suggest that these views of risk are inadequate and that the nature of risk is to some degree idiosyncratic. Like beauty, it is in the eyes of the beholder.

We shall consider games (we prefer this term to gambles) of the form $g=(y, p, z)$ where $p$ is the probability of obtaining $y$ amount of money, otherwise $z$ amount, and $y \geqslant z$. We propose in portfolio theory that such a game is perceived in terms of, and may be characterized by, two parameters, expected value, $E$, and perceived risk, $R$.

${ }^{1}$ The study reported here was supported in part by National Science Foundation Grant GB-6782 to the University of Michigan. We would like to also express our appreciation to David Krantz, J. E. Keith Smith, and Amos Tversky for criticism and much discussion. 
Expected value is equal to the inner product of the outcomes and their respective probabilities, but risk is left essentially undefined.

In this paper we propose a structure for perceived risk and present an experimental test of some of the conditions implied by the structure. Potentially it can lead to the measurement of perceived risk in spite of certain individual variations in the concept of risk.

The approach is to define transformations on these games which permit transforming any one of these games into any other with the same probability of winning. We propose that these transformations induce corresponding transformations on perceived risk. We add another transformation for the multiple play of a game which may also induce a transformation on perceived risk. We propose that the joint effect of these transformations on perceived risk has a particular polynomial form which we test by conjoint measurement.

\section{Construction of Games}

Let $\Omega_{p}$ denote the space of all two-outcome games for which $p$ is the probability of winning $y$ amount of money, otherwise $z$ (with probability $q-1-p$ ), and $y \geqslant z$.

We define the following three sets of transformations on these games:

$$
\begin{aligned}
& A=\left\{a, a^{\prime}, a^{\prime \prime} \cdots\right\} \\
& B=\left\{b, b^{\prime}, b^{\prime \prime} \cdots\right\} \\
& C=\left\{c, c^{\prime}, c^{\prime \prime} \cdots\right\}
\end{aligned}
$$

and

$$
\begin{array}{ll}
a(g)=\left(y+a, p, z-\frac{p}{q} a\right) & \forall a \in A, \\
b(g)=(y+b, p, z+b) & \forall b \in B, \\
c(g)=(y, p, z)^{(c)} & \forall c \in C,
\end{array}
$$

in which $a$ and $b$ are real numbers and $c$ is a nonnegative integer and designates that the game $g$ is played $c$ times independently.

Letting $E(g)$ indicate the expected value of $g$, it is easy to show that $a(g)$ is expectation-preserving and $b(g)$ increments the expectation by the amount $\ell$. That is

$$
\begin{aligned}
& E[a(g)]=E(g), \\
& E[b(g)]=E(G)+b .
\end{aligned}
$$

It is also easy to show that any game $g \in \Omega_{p}$, can be converted by these two 
transformations into any other game $g^{\prime} \in \Omega_{p}$. To transform $g=(y, p, z)$ into $g^{\prime}=\left(y^{\prime}, p, z^{\prime}\right)$ we must have

$$
\begin{aligned}
& y^{\prime}=y+a+l, \\
& z^{\prime}-z-\frac{p}{q} a+l .
\end{aligned}
$$

Solving for $a$ and $b$ we have:

$$
\begin{aligned}
& a=q\left[\left(y^{\prime}-y\right)-\left(z^{\prime}-z\right)\right], \\
& \ddots=p\left(y^{\prime}-y\right)+q\left(z^{\prime}-z\right),
\end{aligned}
$$

and these equations always have a solution.

Transformations $a$ and $b$ are associative and commutative and are always applied before multiple play,

The transformation, $c(g)$, for independent multiple-play, is the convolution of the game $g$ with itself $c$ times. For example, with $e=2$, the game $g$ is transformed into a three-outcome game $g^{\prime}$, with outcome $2 y$ with probability $p^{2}$, outcome $y+z$ with probability $2 p q$, and outcome $2 z$ with probability $q^{2}$, i.e.:

$$
g^{\prime}=\left(2 y, p^{2}, y+z, 2 p q, 2 z, q^{2}\right) \text {. }
$$

Because independent multiple-play accumulates expectation, we have

$$
E[c(g)]={ }^{2} E(g) .
$$

We expect each of these transformations to have an effect on the perceived riskiness of a game, specific to an individual, reflecting his particular conception of what is risk. We also expect that for many, if not most, these effects will be ill-defined and fuzzy. The question arises, then, whether any structure can be found at all in perceived risk and what generality it might have.

We shall use $g_{0}=(0, p, 0)$ as the origin from which all other two-outcome games with the same probability of winning will be generated by means of the transformations $A$ and $B$. With the multiple-play transformation $C$, we have three functions which we will consider to be the three variables of our system. We seek to study the perceived risk structure of this set of games in which these transformations are to be our variables.

For later psychological relevance it is useful to point out that a two-outcome game with fixed $p$ generated from $g_{0}$ by $a \in A$ and $b \in B$ is the game $(a+t, p, t-(p / q) a)$ and for which the expected regret is $q[(a+\ell)-(\ell-(p / q) a)]=a$ and the expected value is $\ell$. Furthermore, the effect of multiple play can be easily seen to multiply both the expected regret and the expected value. 


\section{Alternative Polynomials for Perceived Risk}

We assume the existence of a real valued function, $R$, which assigns a measure of risk $R(g)$, to each game generated from $g_{0}$ by means of the transformations $A, B$, and $C$, and we wish to study the composition function by which this measure depends on these thrce variables. The combination rules to be considered are those analyzed by Krantz and Tversky (1969) in which the variable $r_{2}$ adds to or multiplies $r_{1}$ and the variable $r_{3}$ than either adds to or multiplies the sum or product of $r_{1}$ and $r_{2}$ :

$$
\begin{array}{cl}
r_{1}+r_{2}+r_{3} & \text { (additive model), } \\
\left(r_{1}+r_{2}\right) r_{3} & \text { (distributive model), } \\
r_{1} r_{2}+r_{3} & \text { (dual-distributive model), } \\
r_{1} r_{2} r_{3} & \text { (multiplicative model). }
\end{array}
$$

Because we expect the effect of the $A$ and $B$ transformations to be additive and transformation $C$, the number of plays, to be multiplicative over the joint effect of the other two, we direct our attention mostly to necessary and distinguishing properties of the distributive model.

Every triple $a \in A, b \in B, c \in C$, represents a game, $g \equiv[a, b, c]$, from $g_{0}=(0, p, 0)$.

The representation theorem we seek to establish, then, is the following: there exist real valued functions $\alpha, \beta$, and $\gamma$, defined on $A, B$, and $C$, respectively, such that

$$
g \equiv[a, b, c] \leqslant g^{\prime} \equiv\left[a^{\prime}, b^{\prime}, c^{\prime}\right] \quad \text { iff }[\alpha(a)+\beta(\ell)] \gamma(c) \leqslant\left[\alpha\left(a^{\prime}\right)+\beta\left(\ell^{\prime}\right)\right] \gamma\left(\ell^{\prime}\right)
$$

where $\preccurlyeq \equiv$ an empirical binary relation on perceived risk

$\leqslant \equiv$ the natural weak ordering on real numbers.

Heuristically, $\alpha, \beta$, and $\gamma$ correspond to three psychophysical functions for the subjective effects on perceived risk of the corresponding transformations $A, B$, and $C$, respectively, on games. These mathematical transformations on games are our independent variables in this theory of perceived risk and play a role analogous to that which physical energy does in ordinary sensory psychophysics.

As the transformation $A$ is expectation-preserving it would not be surprising if $\alpha$ were an increasing monotone function of $\alpha$, because almost every candidate for a notion of risk, like variance, maximum possible loss, expected loss, and expected regret all increase with $\alpha$.

The transformation $B$ has no effect on variance or expected regret but maximum loss and expected loss vary inversely with $\ell$, hence one might anticipate that $\beta$ would not be an increasing function of $\ell$.

The transformation $C$ changes all such measures at the same time and in directions which could have opposite effects on perceived risk. 
We are interested, then, in whether perceived risk has the structure of the simple distributive model and, if so, what might be said further about the functions $\alpha, \beta$, and $\gamma$ in individual cases.

\section{Diagnostic Properties}

If we generate a set of games from the Cartesian product $A \times B \times C$ with some suitable fixed number of levels in each factor, and then obtain an empirical rank order of the riskiness of these games, we have a data matrix with a number in each cell representing the rank order of the conjoint effect of some fixed combination of the factors $A, B$, and $C$. There are various properties which this empirical ordering may exhibit which can be used to identify and distinguish the various polynomial models (Krantz and Tversky, 1969). We shall present these and discuss their application to the problem of testing the suitability of the distributive model.

A basic concept that is relevant here is that of independence. If the observed ordering of the joint effect of any subset of the variables at fixed levels of another second (disjoint) subset of the variables is unaffected by the levels at which these latter variables are fixed, all remaining variables held constant, the first subset of variables is said to be independent of the second. In the three variable case, the variable $A$ is independent of $B$ if the ordering induced on $A$ with $B$ and $C$ held constant does not depend on the fixed value of $B$. If a single variable and the joint effect of the remaining variables are mutually independent, then the single variable is called semiaddilive.

Sign-dependence is a more general form of independence. Consider the product of two numbers, $x$ and $y$, and the order induced on the product, $x y$, by fixing the value of $x$. Clearly the ordering thereby induced on $x y$ is not independent of the fixed value for $x$, because if $x$ is negative the ordering on $x y$ is exactly reversed from the ordering on $x y$ if $x$ is positive. Furthermore, if $x-0$ the ordering induced on $x y$ is degenerate. However, the variable $x$ can be partitioned into signed classes (positive, negative, zero) such that the ordering induced on $x y$ is independent of the choice of the value of $x$ except for the "sign" of that value, with reversed sign reversing the ordering and the "zero-sign" producing degenerate ordering.

In the three variable case we say that the factor $A$ is "sign-dependent" on $C$ if the ordering induced on $A$ by fixing $B$ and $C$ is independent of the level of $C$ except for the sign of that level $\left(C^{+}, C^{0}, C^{-}\right)$. For example, in the distributive model of Eq. $9, A$ is independent of $B$ and sign-dependent on $C$. If $C$ has only one sign (positive), however, then $A$ is independent of $C$.

The discussion and subsequent analysis of data, organizes itself conveniently around three sets of properties: (i) those properties which must hold for any of these four polynomial models to obtain; (ii) those properties which distinguish, on the 
one hand, the additive and multiplicative models, from the distributive and dualdistributive models on the other; and (iii) a property which distinguishes between the distributive and the dual-distributive models.

\section{Plane Properties}

The first set of properties might be referred to as "plane" properties because they are necessary conditions for additivity to hold in a plane, by which is meant holding one variable constant, the joint effect of the other two must be additive. Additivity in every plane is a property which these four polynomials have in common provided that all scale values have the same sign. For example, in the distributive model of Eq. 9, holding $r_{2}$ constant and taking logarithms makes the function additive if all scale values are positive, because we would have $\log \left(r_{1}+c\right)+\log r_{3}$. If all scale values were negative, the ordering would be unaffected because the value of the function is unchanged. But if $r_{1}$ were positive and $r_{2}$ were negative, for example, they could cancel and yield a degenerate ordering.

Two necessary conditions for additivity in every plane are: (i) independence in planes, that is, independence of each variable relative to each other variable with the third variable held constant, and (ii) double cancellation in every plane.

\section{Independence in Planes}

Examples of the first condition are the independence of $A$ with respect to $B$ with $C$ held constant; in formal terms:

$$
\begin{gathered}
{[a, b, c] \leqslant\left[a^{\prime}, b, c\right] \quad \text { iff }\left[a, b^{\prime}, c\right] \leqslant\left[a^{\prime}, b^{\prime}, c\right]} \\
\text { for all levels of } A \text { and } B \text { with } C \text { fixed; }
\end{gathered}
$$

and the independence of $B$ with respect to $A$ with $C$ held constant requires that:

$$
[a, b, c] \leqslant\left[a, b^{\prime}, c\right] \quad \text { iff } \quad\left[a^{\prime}, b, c\right] \leqslant\left[a^{\prime}, b^{\prime}, c\right]
$$

for all levels of $A$ and $B$ with $C$ fixed.

A graphical representation of these independence properties on the plane is given in Fig. 1, in which (12.i) is represented by the vertical arrows and (12.ii) is represented

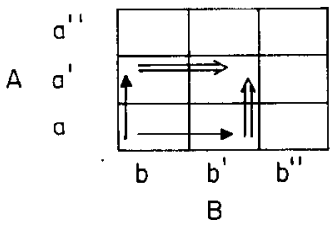

Fig. 1. Mutual independence of $A$ and $B$ in an $A \times B$ plane. 
by the horizontal arrows. The single arrows representing the left side of their respective expressions, the double arrow the right side. Each arrow represents an empirical binary relation with the arrowhead indicating the cell which is at least as risky.

\section{Double Cancellation}

The property of double cancellation is satisfied in the $A \times B$ plane for a fixed level of $C$ iff for all levels of $A \times B$ we have:

$$
\begin{aligned}
{\left[a, b^{\prime}, c\right] } & \leqslant\left[a^{\prime}, b, c\right], \\
\text { and } \quad\left[a^{\prime}, b^{\prime \prime}, c\right] & \leqslant\left[a^{\prime \prime}, b^{\prime}, c\right], \\
\text { imply } \quad\left[a, b^{\prime \prime}, c\right] & \leqslant\left[a^{\prime \prime}, b, c\right] .
\end{aligned}
$$

A graphical representation of double cancellation is given in Fig. 2 in which the single arrows represent the two hypotheses and the double arrow the implication.

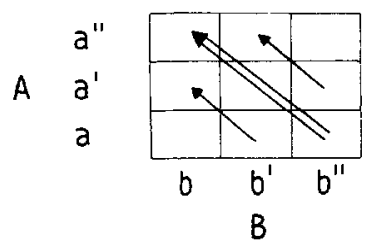

FIG. 2. Double cancellation in an $A \times B$ plane.

In this first set of properties, one variable is held constant and certain properties are observed of the ordering of the joint effect from varying the other two.

The second set of properties pertain to the effect of the level at which the one variable is held constant (or to the effect of the levels at which two of the variables are jointly held constant). Another way of putting it is that the first set of properties are intraplane properties, the second set all involve effects of passing from one plane to another, interplane properties.

\section{Interplane Properties}

These properties serve the purpose of testing whether or not the effect of the variables, taken one at a time and taken two at a time, is additive with the remaining variable(s). Such properties are critical for distinguishing the additive and multiplicative models from the distributive and dual-distributive models.

In the distributive model of Eq. 9 for example, and with the real numbers as the normal domain of all variables, $r_{3}$ should be sign-dependent on $\left(r_{1}+r_{2}\right)$ and, also, $\left(r_{1}+r_{2}\right)$ should be sign-dependent on $r_{3}$. That is, the ordering induced on $r_{3}$ by holding $\left(r_{1}+r_{2}\right)$ constant depends only on whether the value of $\left(r_{1}+r_{2}\right)$ is positive, zero, or negative, and vice versa. If the domain of the three variables is only in the 
positive reals, however, then the above induced orderings are independent and the two subsets of variables, $\left(r_{1}, r_{2}\right)$ and $r_{3}$, are semiadditive. These relations, of course, would also be true if the model were additive or multiplicative.

However, the induced joint ordering of any two other variables, e.g., $\left(r_{1}, r_{3}\right)$ could violate independence under the distributive model, but not under the additive or multiplicative even if the domain of all variables is in the positive reals.

Formally, for our variable $C$ to be independent of the joint effects of $A$ and $B$, we must have for all levels of $A, B$, and $C$,

$$
[a, b, c] \leqslant\left[a, b, c^{\prime}\right] \quad \text { iff } \quad\left[a^{\prime}, b^{\prime}, c\right] \leqslant\left[a^{\prime}, b^{\prime}, c^{\prime}\right]
$$

A graphical representation of this property is presented in Fig. 3 .

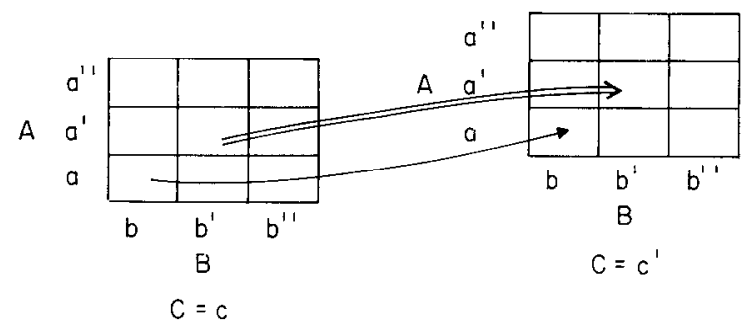

Fig. 3. Independence of $C$ with respect to $A \times B$.

However, for our variables taken one at a time, say $C$, to be independent of the joint effects of the other two, $A \times B$, it is sufficient that $C$ be independent of $A$ and that $C$ be independent of $B$. Therefore, the independence of one variable with respect to the joint effects of the other two is a consequence of intraplane tests.

For example, the independence of $C$ with respect to $A$ with $B$ fixed means that:

$$
[a, b, c] \leqslant\left[a, b, c^{\prime}\right] \Leftrightarrow\left[a^{\prime}, b, c\right] \leqslant\left[a^{\prime}, b, c^{\prime}\right],
$$

and the independence of $C$ with respect to $B$ with $A$ fixed means that:

$$
\left[a^{\prime}, b, c\right] \leqslant\left[a^{\prime}, b, c^{\prime}\right] \Leftrightarrow\left[a^{\prime}, b^{\prime}, c\right] \leqslant\left[a^{\prime}, b^{\prime}, c^{\prime}\right],
$$

and by transitivity we have expression (14). Hence, given that the intraplane properties are satisfied, as required, the interplane properties which remain to be tested for, are the independence of the variables taken two at a time with respect to the remaining variable. Of course, there are three such tests.

For the joint effect of $A \times B$ to be independent of $C$, we must have, for all levels of $A, B$, and $C$,

$$
[a, b, c] \leqslant\left[a^{\prime}, b^{\prime}, c\right] \quad \text { iff } \quad\left[a, b, c^{\prime}\right] \leqslant\left[a^{\prime}, b^{\prime}, c^{\prime}\right] .
$$

A graphical representation of this property is presented in Fig. 4. 


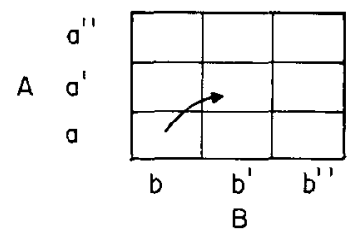

$C=c$

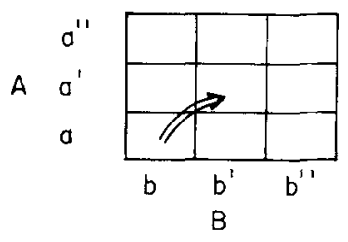

$C=c^{\prime}$

Fig. 4. Independence of $A \times B$ relative to $C$.

If expression (15) holds only if $c, c^{\prime}$ are both elements of $C^{+}$or of $C^{-}$, then $A \times B$ is not independent but is sign-dependent on $C$.

By interchanging the factors $A, B$, and $C$ in expression (15) we have three distinct tests in this set of properties of the interplane variety.

We have now discussed two sets of predictions. The intraplane predictions which must be satisfied if any of the four polynomials (8)-(11) is to obtain; and the interplane predictions, which, if not satisfied as anticipated, reduces attention to the distributive and dual-distributive models. The property, then, which is next of interest, is that which may distinguish between these two models.

\section{Distributive Cancellation}

That distributive cancellation is a necessary condition for the distributive model may be shown as follows. If the distributive decomposition is to hold, then:

$$
\begin{gathered}
{[a, b, c] \leqslant\left[a^{\prime}, b^{\prime}, c^{\prime}\right] \Leftrightarrow\left[\alpha(a)+\beta\left(\ell^{\prime}\right)\right] \gamma(c) \leqslant\left[\alpha\left(a^{\prime}\right)+\beta\left(f^{\prime}\right)\right] \gamma\left(c^{\prime}\right),} \\
{\left[a^{\prime}, b^{\prime}, c\right] \leqslant\left[a^{\prime \prime}, b^{\prime \prime}, c^{\prime}\right] \Leftrightarrow\left[\alpha\left(a^{\prime}\right)+\beta\left(\ell^{\prime}\right)\right] \gamma(c) \leqslant\left[\alpha\left(a^{\prime \prime}\right)+\beta\left(\ell^{\prime \prime}\right)\right] \gamma\left(c^{\prime}\right),} \\
{\left[a^{\prime \prime}, b^{\prime}, c^{\prime}\right] \leqslant\left[a^{\prime}, b, c\right] \Leftrightarrow\left[\alpha\left(a^{\prime \prime}\right)+\beta\left(b^{\prime}\right)\right] \gamma\left(e^{\prime}\right) \leqslant\left[\alpha\left(a^{\prime}\right)+\beta\left(\ell^{\prime}\right)\right] \gamma(c) .}
\end{gathered}
$$

By simple algebra, the right-hand set of inequalities yields:

$$
\left[\alpha(a)+\beta\left(\ell^{\prime}\right)\right] \gamma(c) \leqslant\left[\alpha\left(a^{\prime}\right)+\beta\left(\ell^{\prime \prime}\right)\right] \gamma\left(c^{\prime}\right),
$$

which implies the following empirically testable relation:

$$
\left[a, b^{\prime}, c\right] \leqslant\left[a^{\prime}, b^{\prime \prime}, c^{\prime}\right] .
$$

We may illustrate graphically what is meant by distributive cancellation in the following manner. We represent the two planes of $A \times B$ obtained by fixing $C$ at $C$ and $c^{\prime}$ by the two $3 \times 3$ matrices in Fig. 5 . The three arcs with single headed arrows represent the three empirical inequalities on the left side of (16). The head end of the arrow indicates the cell which has at least as large a joint effect as the cell at the other end. The double-heated arrow represents the empirical inequality given in (17) which is a consequence of (16). 


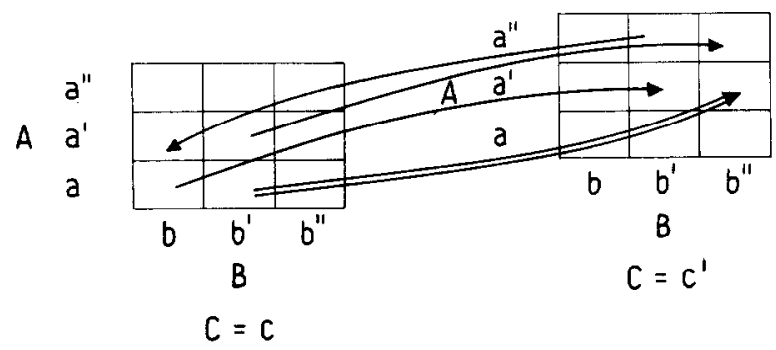

FIG. 5. Distributive cancellation.

Briefly, if one diagonal of a second-order minor at any fixed level of $C$ dominates one diagonal of a second-order minor at any other level of $C$ (or the same level), then the second diagonals of the respective minors cannot have a reverse dominance relation.

That distributive cancellation may be violated by the dual distributive model is shown by the following example in which the numerical arguments are given in the margins and the cell arguments are calculated according to the model $r_{1} r_{2}+r_{3}$ :
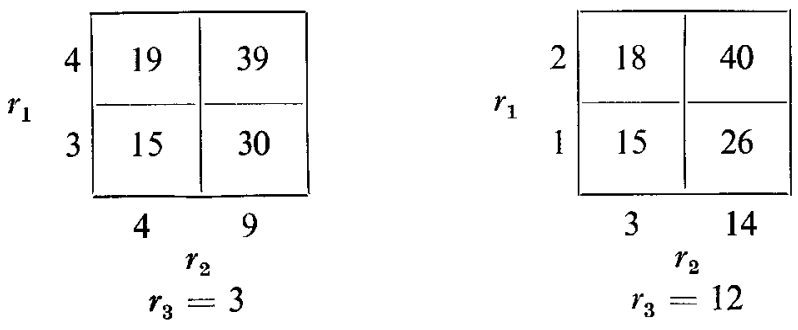

\section{The Experiment}

Subjects. There were originally 36 subjects of whom 28 yielded complete data. There were 21 males and 7 females, all graduate students taking a general mathematical psychology course at the University of Michigan in the winter term of 1968. The data were collected at the beginning of the course and neither conjoint measurement theory nor decision processes had yet been presented.

Stimuli. The basic games were all $p=1 / 2$ and were generated in a $3 \times 3$ matrix by transforming $g_{0}$ as indicated in Table 1 .

The third transformation, multiple play, took on two levels, one and five. So $A \times B \times C=3 \cdot 3 \cdot 2=18$ games in all. For convenience of experimental design, a 19 th game was added, $\left(2.30, \frac{1}{2},-1.30\right)$, played once.

Procedure. The stimuli were arranged in a balanced incomplete block design of 19 sets of 10 games each. In such a design each pair of games is replicated five times. 
TABLE 1

The Basic Games

\begin{tabular}{lccc}
\hline$a^{\prime \prime}=(1.40,-1.40)$ & $\left(1.50, \frac{1}{2},-1.30\right)$ & $\left(1.70, \frac{1}{2},-1.10\right)$ & $\left(1.90, \frac{1}{2},-0.90\right)$ \\
$a^{\prime}=(1.00,-1.00)$ & $\left(1.10, \frac{1}{2},-0.90\right)$ & $\left(1.30, \frac{1}{2},-0.70\right)$ & $\left(1.50, \frac{1}{2},-0.50\right)$ \\
$a=(0.60,-0.60)$ & $\left(0.70, \frac{1}{2},-0.50\right)$ & $\left(0.90, \frac{1}{2},-0.30\right)$ & $\left(1.10, \frac{1}{2},-0.10\right)$ \\
\cline { 2 - 4 } & $b=(0.10,0.10)$ & $b^{\prime}=(0.30,0.30)$ & $b^{\prime \prime}=(0.50,0.50)$ \\
\hline
\end{tabular}

Each game was printed on an IBM card and the cards were correspondingly arranged into 19 decks of 10 cards each.

Each subject was given a complete set of 19 decks and asked to return them 2 days later with each deck arranged in rank order from most to least risky as perceived by the subject. The subjects were asked to rank order each deck independently.

The cards were randomized within each deck and the decks were in an arbitrary order, but no effort was made to insure that the subjects sequentially went through the decks in the order given.

Orderings. For each subject the decomposition (Coombs, 1964, pp. 53-54) of the incomplete blocks provided five replications on each pair of games. These pairwise counts were consolidated into a total rank order of all 19 games in two different ways. One way was to sum for each game the total number of times it was ranked over other stimuli; this ordering is called the total vote count. The second way was to first reduce each pairwise count to the stochastically dominant choice, scored $(1,0)$, and then to sum for each game the total number of other games it was stochastically dominant over; this ordering is called the stochastically dominant ordering.

The stochastically dominant ordering is a vote count ordering in which each pair is weighted equally, consistency matters nought. The total vote count ordering is one in which each vote is weighted equally so in effect, pairs are weighted by their consistency.

Assuming the data to be stimulus comparison data and not preferential choice data in the sense of involving an ideal point (Coombs, 1964), each of the above methods provides an estimate of the rank order stimulus scale for perceived risk. The entire subsequent analysis testing the polynomial model for perception of risk was carried out independently for each of the two rank orders.

\section{REsults}

\section{Consistency and Intransitivity}

The extent to which a subject's pairwise split of the five replications deviates from chance is indicative of his consistency of judgment, and the extent to which weak 
stochastic transitivity is violated is indicative of intransitivity of choice. Inconsistency muddies the water, intransitivity is fatal to the theory.

The degree of a subject's consistency of choice is an important consideration for evaluating his performance relative to a theory. With five replications of a $50 / 50$ choice on each pair the chance distribution of the dominant choice is a folded binomial over 3,4 , and 5 with a mean of 3.44 and a standard deviation of 0.371 . A significant deviation from chance at the .01 level (one-tail test) of the average over 171 pairs is 3.52 or more out of the five replications.

The number out of five replications in the dominant choice, averaged over the 171 pairs, is presented in the first column of Table 2 for each subject. The subjects have been ordered from the most to the least consistent.

As is evident, only one subject, No. 23, fails to deviate significantly from chance, and his performance is almost exactly that expected by chance. This student, when questioned some weeks later, said that he had ordered half the decks on one night and the rest the following night. In retrospect he feared that he had placed them from most to least risky in a deck one night and from least to most the other night, but he was not sure of that. In any event, his level of inconsistency makes his data worthless and represents a failure of the model in that the model says a subject will be able to significantly discriminate differences in risk.

Given a subject's dominant choice on each pair, his intransitivity of choice was evaluated by counting the number of intransitive triples. For $n=19$, the expected number of intransitive triples, if the pairwise choice is 50/50, is 242 and the maximum is 285 . The second column of Table 2 reports the number of intransitive triples for each subject.

The degree of intransitivity appears to closely follow inconsistency. On a scatter diagram the relation is clearly curvilinear. On the whole, intransitivity of choice does not appear to be a problem.

\section{Plane Properties}

Independence in Planes. As a matter of notational convenience in discussing and reporting the independence of one or more variables with respect to one or more others with another held constant a semicolon will be used to indicate "with respect to," and a colon to indicate that what follows it has been held constant. For example, the independence of $A$ with respect to $B$ with $C$ fixed at $c$ would be coded $A ; B: C=c$, the independence of $A$ relative to $B$ in the $C=c$ plane. In this case, $A ; B: C=c$, signifies a comparison of how the effect of the three levels of $A$ are ordered at each of the thrce levcls of $B$ with $C$ fixcd at the level of $c$. Hence, there are three orderings of three elements (cells) which should be the same.

The independence of $A$ with respect to $B$ must be tested at each level of $C, c$, and $c^{\prime}$. So $A ; B: C=c^{\prime}$ also has three orderings of three elements each, all of which should be the same, but not necessarily the same as the orderings for $A ; B: C=c$. 
TABLE 2

Consistency and Intransitivity

\begin{tabular}{|c|c|c|}
\hline Subject no. & Consistency & Intransitivity \\
\hline 4 & 5.000 & 0 \\
\hline 22 & 4.995 & 0 \\
\hline 19 & 4.975 & 0 \\
\hline 20 & 4.960 & 0 \\
\hline 28 & 4.950 & 0 \\
\hline 12 & 4.935 & 0 \\
\hline 18 & 4.925 & 1 \\
\hline 21 & 4.920 & 0 \\
\hline 15 & 4.905 & 0 \\
\hline 35 & 4.845 & 1 \\
\hline 6 & 4.840 & 1 \\
\hline 17 & 4.825 & 2 \\
\hline 5 & 4.800 & 1 \\
\hline 2 & 4.790 & 1 \\
\hline 10 & 4.760 & 3 \\
\hline 30 & 4.750 & 4 \\
\hline 13 & 4.735 & 4 \\
\hline 3 & 4.680 & 1 \\
\hline 26 & 4.650 & 0 \\
\hline 1 & 4.645 & 1 \\
\hline 11 & 4.625 & 3 \\
\hline 36 & 4.605 & 0 \\
\hline 9 & 4.505 & 7 \\
\hline 24 & 4.485 & 14 \\
\hline 25 & 4.350 & 19 \\
\hline 7 & 4.345 & 16 \\
\hline 29 & 4.265 & 16 \\
\hline 23 & 3.430 & 131 \\
\hline
\end{tabular}

Kendall's $\tau$ is linearly related to the proportion of pairwise reversals ${ }^{2}$ between two orderings, $(1-\tau) / 2$. So the average $\tau$ is a measure of the extent to which several orderings agree. An indicator, then, of whether $A$ is independent of $B$ with $C$ held constant, $A ; B: C$, is the average of the two average $\tau$ 's, one for $A ; B: C=c$ and one for $A ; B: C-c^{\prime}$.

In the case of $C ; A: B$ the ordering on the two levels of $C$ are compared at three levels of $A$ for each of the three levels of $B$. So there are nine orderings of two elements. These nine orderings are partitioned into three subsets of three orderings, which should be the same in each subset.

At the heading of each column of Table 3 the particular property of plane inde-

${ }^{2}$ 'Ties were counted one-half. 


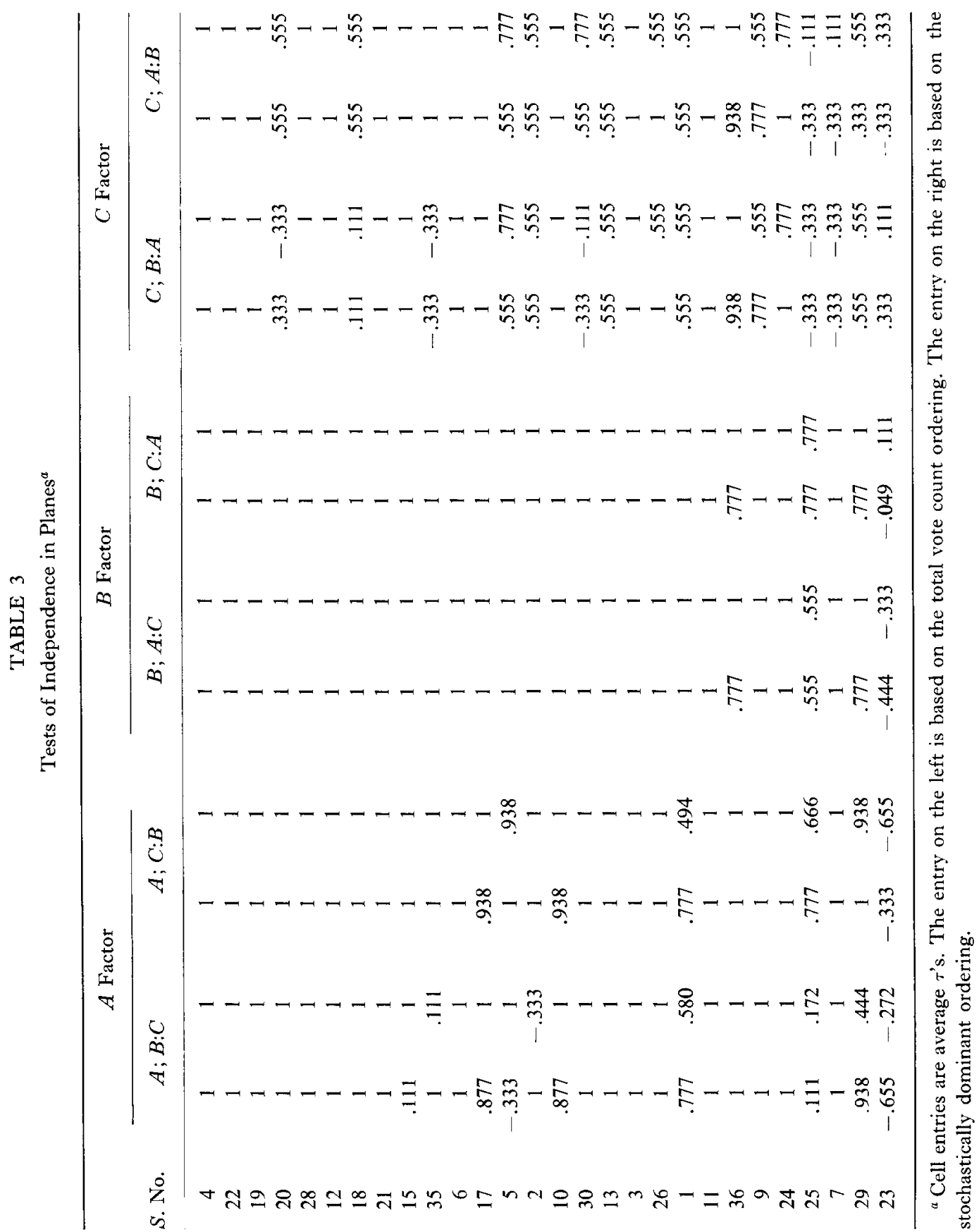


pendence being tested is indicated. The subjects are in the order from most to least consistent (see Table 2) and a cell of the table contains the two average $\tau$ 's for the subject on the test, the average $\tau$ on the left side of the cell is based on the total vote count ordering, and the average $\tau$ on the right side is based on the stochastically dominant ordering. All four polynomials should satisfy this property of independence in every plane, i.e., all $\tau$ 's should be one.

The results based on the different orderings are in substantial agreement and neither ordering seems to be biased in either way with respect to the other. Because the factors do not all have the same number of levels ( 3 for $A$ and $B, 2$ for $C$ ), care must be taken in comparing these average $\tau$ 's. In the case of $C ; A: B$ a reversal on one adjacent pair in one of the three orderings in one subset will reduce the average from one to .555 . Whereas, in the case of the test $A ; B: C$, there are six orderings partitioned into two subsets of three orderings of three elements each. A reversal on one adjacent pair in one of the three orderings in one subset will reduce the average $\tau$ from one to .777 .

In Table 4 we summarize the results of these tests of independence reported in Table 3, by counting the number of subjects out of 28 who had average $\tau$ 's of exactly one and average $\tau$ 's that admit of no more than one pair reversed in one ordering. As is clear from Table 4, factors $A$ and $B$ satisfy independence for almost all subjects, and factor $C$ for at least half the subjects and perhaps three-quarters or more.

Factor $C$ will fail to satisfy independence in this experiment if the transformation from one play to five plays will be perceived as increasing riskiness in the case of some games and decreasing it in others.

Table 4 does not convey the entire story, however, because all six of these properties should hold for each subject. Table 3 shows that there are nine subjects for whom all six properties hold exactly for both basic orderings, i.e., all cell entries are equal to one. There are seven more subjects, $9,10,13,17,24,26,36$, for whom all entries indicate no more than one violation. So for these 16 subjects, we will conclude that all four models are viable in so far as independence in planes is concerned.

\section{Double Cancellation}

Double cancellation should also be satisfied in every plane for any of the simple polynomials to hold. The experimental design, having only two levels of the factor $C$, does not permit testing double cancellation in $A \times C$ or in $B \times C$. So this property is tested in only $A \times B$ at each of the two levels of $C$.

Double cancellation was tested only on those subjects who satisfied plane independence both of $A$ with respect to $B$ and $B$ with respect to $A$. So this property is tested on those subjects who perfectly satisfied both $A ; B: C$ and $B ; A: C$. Hence, the number of subjects on whom the different tests could be made varies. These tests were made, as usual, on the orderings based on the total vote count and on the orderings based on stochastic dominance. 


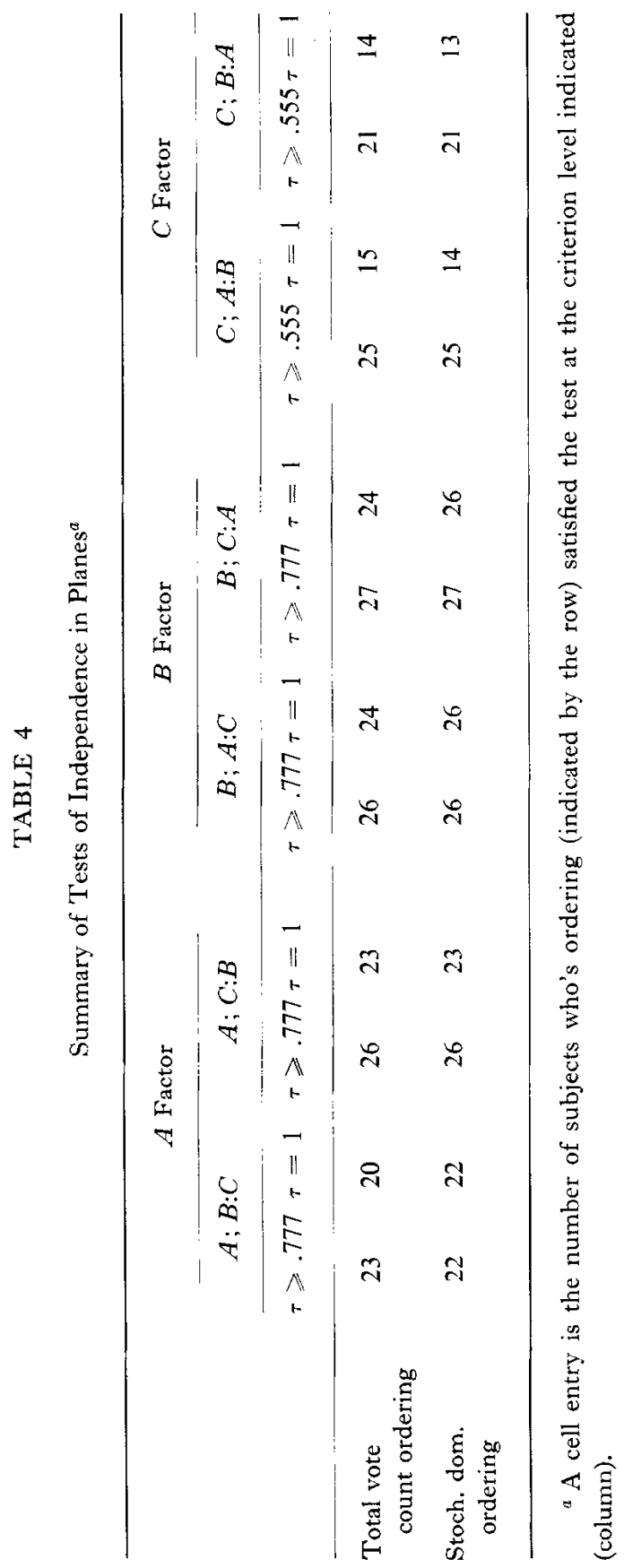


The results are reported in Table 5, where there are three numbers in each cell, the first number is the number of subjects who violated double cancellation; the sccond number is the number of subjects who satisfied all the antecedents for the test and hence a violation could have occured; the third number includes in addition those subjects who satisfied plane independence but for whom the single arrows in Fig. 2 go in the opposite direction and so the test of double cancellation could not be violated.

TABLE 5

Tests of Double Cancellation in $A \times B^{a}$

\begin{tabular}{ccc} 
& $\begin{array}{c}\text { Ordering based on } \\
\text { total vote count }\end{array}$ & $\begin{array}{c}\text { Orderings based on } \\
\text { stochastic dominance }\end{array}$ \\
$A \times B: C=c$ & $1: 18: 21$ & $3: 18: 22$ \\
$A \times B: C=c^{\prime}$ & $0: 16: 20$ & $1: 17: 23$ \\
\hline
\end{tabular}

${ }^{a}$ The three numbers constituting each cell entry are, in turn, the number of subjects who violated double cancellation out of the number of subjects who satisfied the premises for the test out of the number of subjects who satisfied plane independence.

The subject who violated double cancellation in $A \times B: C=c$ in the ordering based on total vote count is number 24 . For the ordering based on stochastic dominance the three subjects who violated it in $A \times B: C=c$ were numbers $3,24,35$, and the one who violated it in $A \times B: C=c^{\prime}$ was number 3. It is to be noted that subjects numbered 3 and 24 were two of the more inconsistent subjects.

\section{Interplane Properties}

The interplane properties involving the independence of each variable taken singly with respect to the joint effect of the other two- $A ; B \times C, B ; A \times C, C ; A \times B-$ are, as we have seen, consequences of independence in their respective planes.

The interplane properties with which we are concerned, then, involve testing for the independence of the joint effects of each pair of variables with respect to the remaining variable (i.e., tests of the form $A \times B ; C$ ) of which there are three.

In principle these tests need to be made only on those subjects who have satisfied the intraplane tests but, because the latter decision is somewhat arbitrary, we report the tests in full.

The three tests differ in the number of elements being ordered, in the number of orderings being compared, and hence in the effect of a single reversal on the average $\tau$. Thus, in testing the independence of $A \times B$ with respect to $C$ we have two orderings of the nine elements in $A \times B$, and if they differ by one reversal of an adjacent pair, the (average) $\tau$ is .944; in both $B \times C$ with respect to $A$ and $A \times C$ 
with respect to $B$, we have three orderings of six elements, so with one reversal of an adjacent pair, the average $\tau$ is .911 .

For the additive and multiplicative models the orderings in each test should all be the same if the scale values within each factor all have the same sign. If the distributive or dual-distributive model obtains, however, only the two orderings of the nine elements of $A \times B$ must be identical. The other two tests may or may not violate independence.

TABLE 6

Interplane Tests of Independence ${ }^{a}$

\begin{tabular}{|c|c|c|c|c|c|c|}
\hline \multirow{2}{*}{$\begin{array}{c}S \text { No. } \\
* * 4\end{array}$} & \multicolumn{2}{|c|}{$A \times B ; C$} & \multicolumn{2}{|c|}{$B \times C ; A$} & \multicolumn{2}{|c|}{$A \times C ; B$} \\
\hline & 1 & 1 & .911 & .911 & .733 & .733 \\
\hline$* * 22$ & 1 & 1 & .911 & .911 & .822 & .822 \\
\hline$* * 19$ & 1 & 1 & .911 & .911 & .822 & .822 \\
\hline 20 & 1 & 1 & .733 & .733 & .377 & .377 \\
\hline$* * 28$ & .944 & 1 & .911 & .911 & .822 & .822 \\
\hline$* * 12$ & 1 & 1 & .911 & .911 & .822 & .822 \\
\hline 18 & .944 & 1 & .911 & .911 & .822 & .822 \\
\hline$* * 21$ & 1 & .929 & .911 & .911 & 1 & 1 \\
\hline 15 & 1 & 1 & 1 & 1 & -.066 & -.066 \\
\hline 35 & .666 & 1 & 1 & 1 & .288 & .200 \\
\hline$* * 6$ & .816 & .783 & I & 1 & 1 & 1 \\
\hline$* 17$ & .986 & .899 & .866 & .866 & .885 & .911 \\
\hline 5 & 1 & 1 & .911 & .977 & .200 & .077 \\
\hline 2 & .944 & .986 & .644 & .644 & .733 & .733 \\
\hline$* 10$ & .957 & .944 & 1 & 1 & .977 & 1 \\
\hline 30 & .957 & .986 & .828 & .887 & .511 & .701 \\
\hline$* 13$ & 1 & .986 & .644 & .644 & .822 & .841 \\
\hline$* * 3$ & .944 & .944 & .733 & .733 & .822 & .822 \\
\hline$* 26$ & 1 & .816 & 1 & .911 & 1 & .911 \\
\hline 1 & .944 & .816 & .911 & .822 & .644 & .571 \\
\hline$* * 11$ & .944 & .929 & 1 & I & .911 & .911 \\
\hline$* 36$ & .944 & 1 & .796 & 1 & .977 & 1 \\
\hline$* 9$ & .971 & .986 & .953 & .885 & .833 & .876 \\
\hline$* 24$ & .888 & .985 & .977 & .977 & 1 & .953 \\
\hline 25 & .777 & .816 & .377 & .476 & -.066 & -.208 \\
\hline 7 & .986 & .944 & .733 & .885 & .733 & .748 \\
\hline 29 & .873 & .857 & .793 & .887 & .718 & .530 \\
\hline 23 & .366 & -.029 & -.233 & -.249 & .066 & .083 \\
\hline $\bar{\tau}$ & .922 & .937 & .823 & .855 & .750 & .784 \\
\hline
\end{tabular}

${ }^{a}$ All entries are average $\tau^{\prime}$ 's. The $\tau$ on the left is based on the total vote count ordering. The $\tau$ on the right is based on the stochastically dominant ordering. 
For example, the test, $B \times C ; A$ may or may not violate independence depending on the levels chosen in the experiment for the three factors, thus,

$$
[\alpha(a)+\beta(\ell)] \gamma(c)<\left[\alpha(a)+\beta\left(\ell^{\prime}\right)\right] \gamma\left(c^{\prime}\right) \quad \text { iff } \quad \alpha(a)<\frac{\beta\left(\ell^{\prime}\right) \gamma\left(c^{\prime}\right)-\beta(t) \gamma(c)}{\gamma(c)-\gamma\left(c^{\prime}\right)},
$$

so, clearly, the ordering on the two cells $[a, b, c]$ and $\left[a, b^{\prime}, c^{\prime}\right]$ is not diagnostic for the model. Another way to help make clear that this test can be violated is to note that the effect of factor $C$ is to magnify the joint effect of $A \times B$. If $A$ increases risk and $B$ decreases it their joint effect may be positive or negative depending on the experimental levels chosen.

The results on the interplane tests are reported in Table 6. At the head of each column the particular property being tested is indicated. The subjects are in the order from most to least consistent and a cell of 'Table 6 contains the two average $\tau$ 's for the subject on that test, the $\tau$ on the left side is based on the total vote count ordering and the one on the right side is based on the stochastically dominant ordering. We have indicated with a double asterisk those subjects who perfectly satisfied all six intraplane tests, and with a single asterisk those subjects who deviated by a single reversal in one ordering.

In Table 7 we summarize the results of these interplane tests of independence. The results are summarized separately for all 28 subjects and for the 16 subjects who satisfied all tests of independence in planes at a minimum level of no more than one pair reversed in one ordering.

TABLE 7

Summary of Interplane Tests of Independence

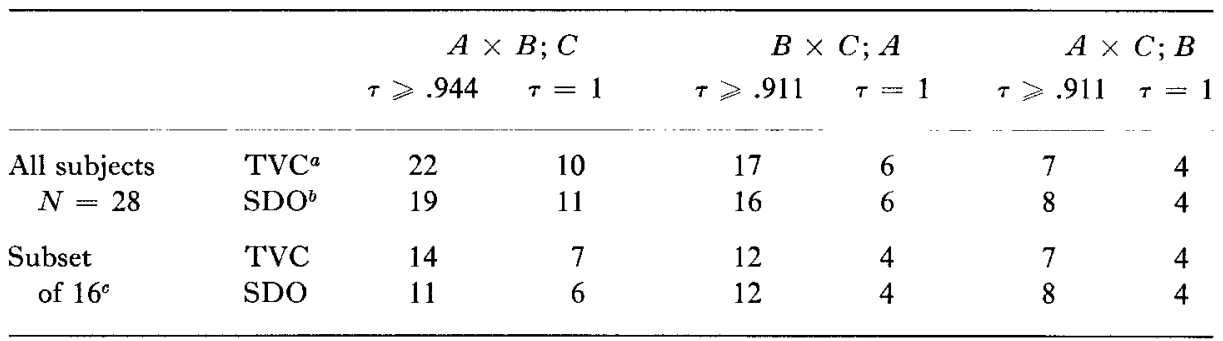

${ }^{a} \mathrm{TVC}=$ the total vote count ordering.

${ }^{b} \mathrm{SDO}=$ the stochastically dominant ordering.

c These are the 16 subjects who satisfied each test of independence in planes according to a criterion of no more than one pair reversed in one ordering.

We see that the independence of $A \times C ; B$ is satisfied by only seven or eight of the subjects, even at the less stringent criterion, and on these grounds we may 
reject the additive model. We may also reject the multiplicative model if we assume that all scale values have the same sign within one factor. We also see from Table 7 that the independence of $A \times B ; C$ is satisfied by most of the subjects, as required by all four models and, in particular, the distributive and dual-distributive. So we turn to the test of distributive cancellation.

\section{Distributive Cancellation}

The number of tests that may be made of this property for each subject depends upon his ordering of the cells in the first place. In this experiment, the number of tests that could be made on a subject varied from 0 to 72 .

For each subject, and for each of the two basic orderings, the number of violations of distributive cancellation and the number of tests it was possible to make on the subject are reported in Table 8 . The subjects are ordered from the most to least consistent.

It appears that the property of distributive cancellation is satisfied, supporting the distributive model. We have not, however, eliminated the dual-distributive model, the property of distributive cancellation could be satisfied by a subset of data satisfying the dual-distributive model. Another cancellation property is required by the dual-distributive model but a larger-scale experiment is required to test it. Our preference for the distributive model over the dual-distributive is not based on evidence from this experiment but is based on arguments presented in the discussion.

\section{Discussion}

While only qualitative comparisons of models have been possible in this experiment there appears to be substantial support for the empirical relational system for perceived risk we seek to capture with the distributive model. The principal negative results are the tests of plane independence of $C$ with respect to $A$ with $B$ fixed and of $C$ with respect to $B$ with $A$ fixed, which were satisfied by only half the subjects at the strict criterion level of $\tau=1$ and by three-quarters or more at the lesser criterion level of no more than one reversal.

As a matter of substantive interest riskiness was perceived without exception to increase as $a$ increased, other things being constant, for 20 of the 28 subjects and most of the time for six more. Riskiness was perceived without exception to decrease as $t$ increased other things being constant, for 26 of the 28 subjects and most of the time for the remaining two. The effect of multiple play is merely to enhance the joint effect of the other two factors, according to the distributive model, and this is supported by the data.

One of the structural conditions given by Krantz and Tversky for the distributive model to hold is that $(A \times B)^{0}$ and $C^{0}$ not be empty, i.e., that there must exist a 
TABLE 8

Testing Distributive Cancellation

\begin{tabular}{|c|c|c|c|c|}
\hline \multirow[b]{2}{*}{$\begin{array}{c}\text { Subject } \\
\text { no. }\end{array}$} & \multicolumn{2}{|c|}{$\begin{array}{l}\text { Total vote count } \\
\text { ordering }\end{array}$} & \multicolumn{2}{|c|}{$\begin{array}{c}\text { Stochastic dominance } \\
\text { ordering }\end{array}$} \\
\hline & $\begin{array}{l}\text { No. of } \\
\text { tests }\end{array}$ & $\begin{array}{l}\text { No. of } \\
\text { violations }\end{array}$ & $\begin{array}{l}\text { No. of } \\
\text { tests }\end{array}$ & $\begin{array}{l}\text { No. of } \\
\text { violations }\end{array}$ \\
\hline 4 & 24 & 0 & 24 & 0 \\
\hline 22 & 24 & 0 & 24 & 0 \\
\hline 19 & 24 & 0 & 24 & 0 \\
\hline 20 & 60 & 0 & 56 & 0 \\
\hline 28 & 24 & 0 & 24 & 0 \\
\hline 12 & 24 & 0 & 24 & 0 \\
\hline 18 & 50 & 0 & 60 & 0 \\
\hline 21 & 0 & 0 & 0 & 0 \\
\hline 15 & 5 & 0 & 5 & 0 \\
\hline 35 & 0 & 0 & 0 & 0 \\
\hline 6 & 0 & 0 & 0 & 0 \\
\hline 17 & 34 & 0 & 60 & 0 \\
\hline 5 & 8 & 1 & 8 & 1 \\
\hline 2 & 48 & 0 & 52 & 0 \\
\hline 10 & 8 & 0 & 8 & 0 \\
\hline 30 & 56 & 0 & 50 & 1 \\
\hline 13 & 54 & 2 & 46 & 1 \\
\hline 3 & 33 & 1 & 38 & 2 \\
\hline 26 & 44 & 0 & 46 & 0 \\
\hline 1 & 58 & 0 & 40 & 0 \\
\hline 11 & 20 & 0 & 8 & 0 \\
\hline 36 & 56 & 0 & 52 & 0 \\
\hline 9 & 60 & 7 & 53 & 2 \\
\hline 24 & 29 & 0 & 47 & 3 \\
\hline 25 & 33 & 5 & 19 & 3 \\
\hline 7 & 72 & 4 & 59 & 5 \\
\hline 29 & 35 & 3 & 15 & 0 \\
\hline 23 & 0 & 0 & 1 & 0 \\
\hline Av. & 31.5 & 0.82 & 30.1 & 0.64 \\
\hline
\end{tabular}

level of $A \times B$ for which the ordering induced on $C$ is degenerate, and a level of $C$ for which the ordering induced on $A \times B$ is degenerate.

We would argue that $g_{0}=(0, p, 0)$ in $A \times B$ induces a degenerate ordering on $C$ in that this game is perceived to remain unchanged in risk no matter how frequently it is played, so $g_{0} \in(A \times B)^{0}$. We would also argue that all games in $A \times B$ played zero times are reduced to equal risk, so $c=0 \in C^{0}$. While these properties are not 
strictly necessary they reinforce our view that the distributive model is appropriate and the dual-distributive model is not.

The transformations $A, B, C$ investigated here are not the only ones possible; there are alternatives to these and there are additional transformations to be included. Obvious ones are a transformation on the probabilities and a transformation that introduces dependent multiple play (in contrast to $C$ ). It is not feasible at this time to study five variable polynomials by conjoint measurement methods but different subsets of three could be studied in a manner similar to this experiment.

\section{REFERENCES}

Coombs, C. H. Theory of data. New York: Wiley, 1964.

Coombs, C. H. AND Huang, L. C. A portfolio theory of risk preference. Michigan Mathematical Psychology Program Technical Report MMPP 68-5, University of Michigan, 1968.

CoOmbs, C. H., AND MEYer, D. E. Risk-preference in coin-toss games. Journal of Mathematical Psychology, 1969, 6, 514-527.

Coombs, C. H., AND Pruitt, D. G. Components of risk in decision making: Probability and variance preferences. Journal of Experimental Psychology, 1960, 60, 265-277.

Coomds, C. H., AND Pruitt, D. G. Some characteristics of choice behavior in risky situations. Annals of the New York Academy of Sciences, 1961, 69, 784-794.

Krantz, D. H., And Tversky, A., in preparation, 1969. See also: Krantz, D. H. A survey of measurement theory. In Lectures in applied mathematics, Vol. 12: Mathematics of the Decision Sciences, part 2. Providence, R. I.: Amer. Math. Soc., 1967. Pp. 314-350.

Markowitz, H. M. Portfolio selection. New York: Wiley, 1959.

ROYDEN, H. L., SupPeS, P., AND WALSH, K. A model for the experimental measurement of the utility of gambling. Behavioral Science, 1959, 4, 11-18.

ReCEIvED: March 12, 1969 